Western University

Scholarship@Western

Department of Economics Research Reports

Economics Working Papers Archive

1984

\title{
Estimation and Testing in a Regression Model with Spherically Symmetric Errors
}

Aman Ullah

Victoria Zinde-Walsh

Follow this and additional works at: https://ir.lib.uwo.ca/economicsresrpt

Part of the Economics Commons

Citation of this paper:

Ullah, Aman, Victoria Zinde-Walsh. "Estimation and Testing in a Regression Model with Spherically Symmetric Errors." Department of Economics Research Reports, 8422. London, ON: Department of Economics, University of Western Ontario (1984). 
ISSN :

0318-725x

ISBN : $\quad 0-7714-0604-5$

\section{RESEARCH REPORT 8422 \\ ESTIMATION AND TESTING IN A \\ REGRESSION MODEL WITH SPHERICALLY SYMMETRIC ERRORS \\ by \\ Aman U1lah* and Victoria Zinde-Walsh}

Department of Economics Library

November, 1984

AB STRACT

University of Western Ontario

In this paper we show that both the restricted and unrestricted likelihood estimators of the error variance, obtained under normality, are not in general robust against the spherical distribution of errors. Thus, care should be taken in interpreting the residual variances in the applied work. The robustness of Rao's score, Wald's and the likelihood ratio tests, are also analyzed.

*The research support from the NSERC to A. U11ah is gratefully acknowledged. The authors are grateful to B. K. Sinha and R. S. Singh for useful discussions and suggestions. 
ESTIMATION AND TESIING IN A REGRESSION MODEL

WITH SPHERICALIY SYMMETRIC ERRORS

by
Aman UIlah and Victoria Zinde-Walsh

1. INTRODUCTION

In this paper we consider the problem of estimation and testing in the context of a regression model in which errors follow a spherically symmetric distribution. It has been shown that the maximum likelihood (ML) estimator of the regression coefficients under normal errors is the same as derived under spherically symmetric errors. However, the ML estimator of the error variance under normal distribution is not the same as obtained under the spherical distribution of errors. Finally, it has been shown that the exact likelihood ratio. (IR) test, Rao's Score (RS) test and Wald's (W) test for testing restrictions are robust against the spherical distribution of errors. In the special case of miltivariate-t errors, the results compare with those in U1lah and Zinde-Walsh (1983).

\section{THE MAIN RESULTS}

Let us consider the regression model

(2.1) $\quad y=x \beta+u$

where $y$ is an $n \times 1$ vector of the dependent variable, $X$ is an $n \times p$ matrix of non-stochastic exogenous variables, $B$ is a $\mathrm{p} \times 1$ vector of unknown parameters and $u$ is an $n \times 1$ error vector. 
It is known that if $\mathrm{u}$ is distributed as multivariate normal with mean vector zero and variance-covariance matrix $\sigma^{2} \mathrm{I}$, then the maximum likelihood (ML) estimators for $\beta$ and $\sigma^{2}$, respectively, are given by

$$
(2.2) \quad b=\left(x^{\prime} x\right)^{-1} x^{\prime} y \quad \text { and } \quad s^{2}=\frac{1}{n}(y-X b)^{\prime}(y-X b)
$$

Also, for testing $H_{0}: R \beta=r$ against $H_{1}: R \beta \neq r$, where $R$ is an $m \times p$ constant matrix of rank $m$, and $r$ is an $m \times 1$ vector of constants, the $I R$ test criterion turns out to be

$$
I R=-2 \log l, \text { with } l=\max _{R \beta=r, \sigma^{2}} L\left(\beta, \sigma^{2}\right) / \max _{\beta, \sigma^{2}} L\left(\beta, \sigma^{2}\right)=\left(s^{2} / \hat{\sigma}^{2}\right)^{n / 2} .
$$

in which we have used the restricted ML estimators of $\sigma^{2}$ and $\beta$ given, respectively, as

(2.4) $\quad \hat{\sigma}^{2}=\frac{1}{n}(y-X \hat{\beta})^{\prime}(y-X \hat{\beta})$ and $\hat{\beta}=b-\left(X^{\prime} X\right)^{-1} R^{\prime}\left[R\left(X^{\prime} X\right)^{-1} R^{\prime}\right]^{-1}(R b-r)$.

Likewise, the RS and $W$ test statistics are, respectively, given by

$$
R S=(R b-r)^{\prime}\left[R\left(X^{\prime} X\right)^{-1} R^{\prime}\right]^{-1}(R b-r) / \hat{\sigma}^{2} \text { and } W=\frac{\hat{\sigma}^{2}}{2} R S
$$

We now consider the class of spherically symmetric distributions

$$
\mathrm{f}(\mathrm{u})=\sigma^{-\mathrm{n}} \phi\left(\frac{\mathrm{u}^{\prime} \mathrm{u}}{\sigma^{2}}\right)=\sigma^{-\mathrm{n}} \phi(w), \quad w=\frac{\mathrm{u}^{\prime} \mathrm{u}}{\sigma^{2}}
$$

where $\phi(w)$ is a decreasing function on $[0, \infty)$. Note that both the multivariate normal and the student-t are members of the class of distributions in (2.6). We introduce $b_{\phi}, s_{\phi}^{2}$; and $\hat{\beta}_{\phi}, \hat{\sigma}_{\phi}^{2}$ to represent the unrestricted and restricted ML estimators, respectively, of $\beta$ and $\sigma^{2}$ under the spherically 
symmetric distributions in (2.6). Similarly, $L R_{\phi}, \mathrm{W}_{\phi}$ and $\mathrm{RS}_{\phi}$ will represent $I R, W$ and $R S$ test statistics under $(2.6)$.

Next, we consider ${ }^{w} \phi$ as the unique solution for $w$ in the equation

(2.7) $\quad \frac{n}{2}+w \frac{\phi^{\prime}(w)}{\phi(w)}=0$

where $\phi^{\prime}(w)$ is the first derivative of $\phi(w)$. A sufficient condition for the existence of a unique solution $w_{\phi}$ would be $\phi^{\prime \prime}(w) \phi(w)-\phi^{\prime 2}(w) \leq 0$, that is the concavity of $\log \phi(w)$.

The main results of the paper can then be stated in the following Theorems:

THEOREM 1: Under (2.6), the unrestricted and restricted ML estimators respectively, of the parameters $\beta$ and $\sigma^{2}$ are given by

$$
b_{\phi}=b \quad, \quad s_{\phi}^{2}=\frac{1}{w_{\phi}}(y-X b)^{\prime}(y-X b)
$$

and

$$
\text { (2.9) } \quad \hat{\beta}_{\phi}=\hat{\beta} \quad, \quad \hat{\sigma}_{\phi}^{2}=\frac{1}{w_{\phi}}(y-x \hat{\beta})^{\prime}(y-x \hat{\beta})
$$

where $b$ and $\hat{B}$ are as given in (2.2) and (2.4), respectively, and ${ }^{\phi}$ is the unique solution of $w$ in $(2.7)$.

PROOF: Using (2.6), write the log of the likelihood function for the parameters of the model $\mathrm{y}=\mathrm{X} \beta+\mathrm{u}$ as

$$
\log L\left(\beta, \sigma^{2}\right)=-\frac{n}{2} \log \sigma^{2}+\log \phi(w)=f(y)
$$

where $w=(y-X \beta)^{\prime}(y-X \beta) / \sigma^{2}=u^{\prime} u / \sigma^{2}$. The first-order conditions for the 
maximization of $\log I\left(\beta, \sigma^{2}\right)$ are

(2.11) $\frac{\partial}{\partial \beta} \log I\left(\beta, \sigma^{2}\right)=-\frac{2 \phi^{\prime}(w)}{\phi(w)} \frac{x^{\prime}(y-x \beta)}{\sigma^{2}}=0$

(2.12) $\frac{\partial}{\partial \sigma^{2}} \log L\left(\beta, \sigma^{2}\right)=-\frac{n}{2 \sigma^{2}}-\frac{w}{\sigma^{2}} \frac{\phi^{\prime}(w)}{\phi(w)}=0$.

It is clear from $(2.11)$ that the unrestricted ML estimator of $\beta$ is $b_{\phi}=b$ as given in $(2.2)$ and $(2.8)$. Further, from (2.12) we get

$$
\phi(w)+\frac{2}{n} w \phi^{\prime}(w)=0
$$

as given in $(2.7)$. Let $w_{\phi}=(y-X \beta)^{\prime}(y-X \beta) / \sigma^{2}$ be the only solution of this equation for which the $\log L\left(\beta, \sigma^{2}\right)$ is at maximum. Then the MLl estimator of $\sigma^{2}$ is

$$
s_{\phi}^{2}=\frac{1}{w_{\phi}}\left(y-X b_{\phi}\right)^{\prime}\left(y-X b_{\phi}\right)=\frac{1}{w_{\phi}}(y-X b)^{\prime}(y-X b)
$$

as given in $(2.8)$.

For the restricted $M L$ estimators of $\beta$ and $\sigma^{2}$ we maximize $H=\log L\left(\beta, \sigma^{2}\right)$ $+\lambda(r-R \beta)$ where $\lambda$ is the vector of Lagrangian coefficients. The first-order conditions are

$$
\frac{\partial H}{\partial \beta}=-\frac{2 \phi^{\prime}(w)}{\phi^{\prime}(w)} \frac{X^{\prime}(y-X \beta)}{\sigma^{2}}-\lambda R=0
$$

$$
\frac{\partial \mathrm{H}}{\partial \sigma^{2}}=-\frac{\mathrm{n}}{2 \sigma^{2}}-\frac{w}{\sigma^{2}} \frac{\phi^{\prime}(w)}{\phi(w)}=0, \quad \frac{\partial \mathrm{H}}{\partial \lambda}=r-R \beta=0 \text {. }
$$

The solutions for $\beta$ and $\sigma^{2}$ from $(2.14)$ and $(2.15)$ can then be verified to be as given in (2.9). 
THEOREM 2: Under $(2.6)$, the IR, RS and W test statistics for testing

$H_{0}: R \beta=r$ against $H_{7}: R \beta \neq r$ are given by

(2.16) $\quad L R_{\phi}=I R, \quad R_{\phi}=c_{\phi}^{-1} \delta \phi S \quad$ and $\quad W_{\phi}=c_{\phi} \delta W^{W}$

where $I R, R S$ and $W$ are as given in (2.3) and (2.5), respectively, $c_{\phi}$ is a constant whose value depends on $\phi$, and $\delta_{\phi}=w_{\phi} / \mathrm{n}$.

PROOF: Note that the likelihood function for $\beta$ and $\sigma^{2}$ is

$$
L\left(\beta, \sigma^{2}\right)=\sigma^{-n} \phi(w)=\sigma^{-n} \phi\left[\frac{(y-x \beta)^{\prime}(y-x \beta)}{\sigma^{2}}\right] .
$$

Thus, using the results in $(2.8)$ and $(2.9)$

$$
\max _{\beta, \sigma^{2}} I\left(\beta, \sigma^{2}\right)=s_{\phi}^{-n} \phi\left(w_{\phi}\right)
$$

$$
\max _{R \beta=r, \sigma^{2}} L\left(\beta, \sigma^{2}\right)=\hat{\sigma}_{\phi}^{-n} \phi\left(w_{\phi}\right) .
$$

It is then obvious that $I R_{\phi}=I R$ as given in (2.16).

Next, we derive the RS test statistic. For this, we first obtain the second-order derivatives of $\log L\left(\beta, \sigma^{2}\right)$ in $(2.10)$. These are

$$
\frac{\partial^{2}}{\partial \beta \partial \beta^{\prime}} \log L\left(\beta, \sigma^{2}\right)=\frac{2 \phi^{\prime}(w)}{\phi(w)} \frac{x^{\prime} x}{\sigma^{2}}+4\left[\frac{\phi^{\prime \prime}(w)}{\phi(w)}-\left(\frac{\phi^{\prime}(w)}{\phi(w)}\right)^{2}\right] \frac{x^{\prime} u u^{\prime} x}{\sigma^{4}}
$$

$$
\frac{\partial^{2}}{\partial \beta \partial \sigma^{2}} \log L\left(\beta, \sigma^{2}\right)=\frac{2}{\sigma^{4}}\left[\frac{\phi^{\prime}(w)}{\phi(w)}+w\left(\frac{\phi^{\prime \prime}(w)}{\phi(w)}-\left(\frac{\phi^{\prime}(w)}{\phi(w)}\right)^{2}\right)\right] x^{\prime} u
$$

where $\phi^{\prime \prime}(w)$ is the second derivative of $\phi(w)$.

Now notice that the right-hand side of $(2.21)$ is an odd function of $\mathrm{u}=\mathrm{y}-\mathrm{X \beta}$. Therefore 
(2.22) $\quad \mathrm{E} \frac{\partial^{2}}{\partial \beta \partial \sigma^{2}} \log L\left(\beta, \sigma^{2}\right)=0$.

Further, we observe that

$$
E \frac{\phi^{\prime}(w)}{\phi(w)}=\int_{u} \frac{\phi^{\prime}(w)}{\phi(w)} \sigma^{-n} \phi(w) d u=\sigma^{-n} \int_{u} \phi^{\prime}(w) d u=c_{1 \phi}
$$

$$
\begin{aligned}
& \mathrm{E}\left(\frac{\phi^{\prime}(w)}{\phi(w)}\right)^{2} \frac{u u^{\prime}}{\sigma^{2}}=\sigma^{-n} \int_{u}\left(\frac{\phi^{\prime}(w)}{\phi(w)}\right)^{2} \frac{u u^{\prime}}{\sigma^{2}} \phi(w) d u=c_{2 \phi^{I}} \\
& E \frac{\phi^{\prime \prime}(w)}{\phi(w)} \frac{u u^{\prime}}{\sigma^{2}}=\sigma^{-n} \int_{u} \phi^{\prime \prime}(w) \frac{u u^{\prime}}{\sigma^{2}} d u=c_{3 \phi^{I}}
\end{aligned}
$$

where $c_{1 \phi}, c_{2 \phi}$ and $c_{3 \phi}$ are constants whose values depend on $\phi$.

Thus, using (2 .23)

(2.24) $\quad E\left[-\frac{\partial^{2}}{\partial \beta \partial \beta^{\prime}} \log L\left(\beta, \sigma^{2}\right)\right]=c_{\phi} \frac{X^{\prime} X}{\sigma^{2}}=I(\beta)$

where

$$
c_{\phi}=4\left(c_{2 \phi}-c_{3 \phi}\right)-2 c_{1 \phi}
$$

whose values depend on $\phi$ (see $(2.23)$ ) and $I(\beta)$ represents the information matrix of $\beta$. Further, because of (2.22), the information matrix of $\beta$ and $\sigma^{2}$, $I\left(\beta, \sigma^{2}\right)$ will be a block diagonal matrix. Therefore, the RS test statistic can be written as

$$
\text { (2.26) } \quad R S_{\phi}=\hat{\mathrm{d}}^{\prime} I^{-1}(\hat{\beta}) \hat{\mathrm{d}}
$$

where $I(\hat{\beta})$ and $\hat{d}$ are $I(\beta)$ and $\partial \log L\left(\beta, \sigma^{2}\right) / \partial \beta$, respectively, evaluated at the restricted estimators $\beta=\hat{\beta}_{\phi}=\hat{\beta}$ and $\sigma^{2}=\hat{\sigma}_{\phi}^{2}$. These are $\hat{d}=x^{\prime}(y-x \hat{\beta}) / \hat{\sigma}_{\phi}^{2}$ and $I(\hat{\beta})=c_{\phi} \hat{\sigma}_{\phi}^{-2} X^{\prime} X$. The result in (2.16) for $\mathrm{RS}_{\phi}$ is then obvious, by noting that $(y-X \hat{\beta})^{\prime} X\left(X^{\prime} X\right)^{-1} X^{\prime}(y-X \hat{\beta})=(R b-r)^{\prime}\left[R\left(X^{\prime} X\right)^{-1} R^{\prime}\right]^{-1}(R b-r)$. 
Finally, consider the $W$ statistic

$$
W_{\phi}=(R b-r)^{\prime}\left[R I^{-1}(b) R^{\prime}\right]^{-1}(R b-r) ;
$$

where $I(b)=I\left(b_{\phi}\right)=c_{\phi} X^{\prime} X / s_{\phi^{-}}^{2}$ Substituting this in $(2.27)$ the result in $(2.16)$ for $W_{\varnothing}$ follows.

Q.E.D.

\subsection{Remarks on the Results}

The following remarks are based on the results in the Theorems.

1. The results in Theorm 1 show that $b_{\phi}=b$ and $\hat{\beta}_{\phi}=\hat{\beta}_{\text {. Thus both }}$

the unrestricted and restricted $\mathrm{ML}$ estimators of $\beta$ are numerically robust against the spherically symmetric distribution of errors. However, $s_{\phi}^{2} \neq s^{2}$ and $\hat{\sigma}_{\phi}^{2} \neq \hat{\sigma}^{2}$ which imply that the restricted as well as unrestricted estimators of $\sigma^{2}$ are not in general robust. For example, if $u$ follows a miltivariate student-t distribution with mean zero and variance $\sigma^{2} I$, then it can easily be verified that $s_{\phi}^{2}=\gamma(\gamma-2)^{-1} s^{2} \neq s^{2}$ and $\hat{\sigma}_{\phi}^{2}=\gamma(\gamma-2)^{-1} \hat{\sigma}^{2} \notin \hat{\sigma}^{2}$. Further, if

$$
\begin{aligned}
f(u) & =c\left(\sigma^{2}\right)^{\frac{-n}{2}} \exp \left[\frac{1}{2} \frac{u^{\prime} u}{\sigma^{2}}\right] & & \text { for } \frac{u^{\prime} u}{\sigma^{\prime}} \leq \omega \\
& =0 & & \text { for } \frac{u^{\prime} u}{\sigma^{2}}>\omega,
\end{aligned}
$$

where $c$ is a constant and $\omega<n$, then the ML estimator of $\sigma^{2}$ is $s_{\varnothing}^{2}=\frac{1}{\omega} \hat{u}^{\prime} \hat{u} \neq s^{2}$. Similarly, if we consider a distribution

$$
f(u)=c\left(\sigma^{2}\right)^{\frac{-n}{2}}\left(1+\frac{u^{\prime} u}{\sigma^{2}}\right)^{-k}, 2 k>n,
$$

then $s_{\phi}^{2}=\left(\frac{2 k}{n}-1\right) \hat{u}^{\prime} \hat{u}$, which will coincide with $s^{2}$ only in the case where $\mathrm{k}=\mathrm{n}+1 / 2$.

2. The result in Theorem 2 shows that although the LR test is numerically robust $\left(L R_{\phi}=L R\right.$ ) the $R S$ and $W$ tests are not numerically robust $\left(\mathrm{RS} \phi \neq \mathrm{RS}\right.$ and $\mathrm{W}_{\varnothing} \neq \mathrm{W}$ ) against the spherically symmetric distribution of errors. 
This result is similar to one in U1lah and Zinde-Walsh (1983) in the multivariate-t case.

Next, we observe that, under $\mathrm{H}_{0}$, all the $\mathrm{LR} \mathrm{R}_{\phi}, \mathrm{RS} \mathrm{S}_{\varnothing}$ and $\mathrm{W}_{\phi}$ tests are monotonic functions of central $F=q($ RRSS - URSS) $/$ mURSS $\sim f(m, q)$ where RRSS $=(y-x \hat{\beta})^{\prime}(y-x \hat{\beta})$, URSS $=(y-x b)^{\prime}(y-x b)$ and $q=n-p$. That the Fstatistic is distributed as a central $\mathrm{F}$ under $\mathrm{H}_{\mathrm{O}}$ whatever $\phi(\omega)$ may be follows from the result of David (1977). The implication of this result is that the test criterion for the normal distribution of errors remains the same under the spherically symmetric distribution of errors. That is we reject $\mathrm{H}_{\mathrm{O}}$ if

$$
\begin{aligned}
& \mathrm{LR}_{\phi}>\mathrm{LR}_{\phi}^{\alpha} \text { or } \quad \mathrm{LR}>\mathrm{LR}^{\alpha} \\
& \mathrm{RS}_{\phi}=\mathrm{c}_{\phi}^{-1} \delta \delta_{\phi}^{\mathrm{RS}}>\mathrm{c}_{\phi}^{-1} \delta_{\phi}^{\mathrm{RS}}{ }^{\alpha} \text { or } \mathrm{RS}>\mathrm{RS}^{\alpha} \\
& \mathrm{W}_{\phi}=\mathrm{c}_{\phi} \delta \delta^{\mathrm{W}}>\mathrm{c}_{\phi} \delta_{\phi} \mathrm{W}^{\alpha} \quad \text { or } \mathrm{W}>\mathrm{W}^{\alpha}
\end{aligned}
$$

where $\mathrm{LR}^{\alpha}, \mathrm{RS}^{\alpha}$ and $\mathrm{W}^{\alpha}$ are the $\alpha \%$ critical points of LR, RS and $\mathrm{W}$, respectively.

From the above findings it is clear that although $\mathrm{RS}_{\phi}$ and $\mathrm{W}_{\phi}$ are not numerically robust they are robust for testing purposes. This latter robustness we call inference robustness. Note that the IR test achieves both inference and numerical robustness. These distinct ideas of robustness were not explicitly stated in Ullah and Zinde-Walsh (1983).

The inequality results for $\mathrm{LR}_{\phi}, \mathrm{RS}_{\phi}$ and $\mathrm{W}_{\phi}$ can be developed for a given $\varnothing$ as in U1lah and Zinde-Walsh. Also, as in the multivariate-t case of U1lah and Zinde-Walsh if the large sample critical value based on chisquare is used, then the tests $L R_{\phi}, R_{\phi}$ and $\mathrm{W}_{\phi}$ will differ with respect to their sizes and powers in small samples and there may be conflict between their conclusions. 


\section{References}

David, A. P., "Spherical Matrix Distributions and a Multivariate Model," J. Roy. Statistic. Soc., Sec. B., 1977, 39, 254-261.

U1lah, A. and V. Zinde-Wa1sh, "On the Robustness of LM, LR and W Tests in Regression Models," Research Report 8316, University of Western Ontario, 1983. Forthcoming in Econometrica, July 1984. 